\title{
Near infrared variability of Sgr A* - spectral index measurements
}

\author{
G. Witzel ${ }^{1}$, M. Morris ${ }^{1}$, A. Ghez ${ }^{1}$, L. Meyer ${ }^{1}$, \\ E. Becklin ${ }^{1}$, K. Matthews ${ }^{2}$, J. R. Lu ${ }^{3}$, \\ T. Do ${ }^{4}$ and R. Campbell ${ }^{5}$ \\ ${ }^{1}$ Dep. of Physics and Astronomy, University of California Los Angeles (UCLA), 465 Portola \\ Plaza, Los Angeles, CA 90095, USA \\ email: witzel@astro.ucla.edu \\ ${ }^{2}$ Caltech, Pasadena, USA $;{ }^{3}$ Institute for Astronomy, University of Hawaii, USA \\ ${ }^{4}$ Dunlap Institute for Astronomy and Astrophysics, University of Toronto, Canada \\ ${ }^{5}$ Keck Observatory, Kamuela, Hawaii, USA
}

\begin{abstract}
We discuss observations of Sagittarius A* with NACO@VLT in $K$-band and recent synchronous observations with NIRC2@Keck II and OSIRIS@Keck I in $L^{\prime}$-band and $H$-band, respectively. The variability of Sagittarius $A^{*}$ in the near infrared is a continuous one-state process that can be described by a pure red-noise process having a timescale of a few hours. We describe this process and its properties in detail. Our newest observations with the Keck telescopes represent the first truly synchronous high cadence data set to test for time variability of the spectral index within the near infrared. We discovered a time-variable spectral index that might be interpreted as a time lag of the $L^{\prime}$-band with respect to the $H$-band.
\end{abstract}

Keywords. Galaxy: center — black hole physics — radiation mechanisms: general — methods: statistical

\section{Introduction}

Sagittarius A* (Sgr A*), the electromagnetic counterpart of the supermassive black hole in the center of our Milky Way, is a highly variable source in the near infrared (NIR). While Sgr A* often is not always bright enough to be detected, about half the time it shows outbursts that are significant with respect to the detection limit of the VLT.

The origin and the characteristics of this variability have been subject to many, partially controversial discussions in the literature (see overviews in Genzel et al. 2010 and Morris et al. 2012). The short timescales (10 minutes) of flux density variations of up to a factor of 30 as well as the redness of the source compared to the stellar environment (Hornstein et al. 2007) and the high linear polarization degree of the intrinsic radiation (Eckart et al. 2006) are indicative of a synchrotron radiation process. Genzel et al. (2003), Eckart et al. (2006) and Meyer et al. (2006) interpreted the short-time structure of the variability as a quasi-periodic signature of orbital motion close to the innermost stable orbit of the black hole, while Do et al. (2009), Meyer et al. (2009) and Witzel et al. (2012) emphasize the red-noise character of the variability process that seems to be sufficient to fully explain the variations of the source at all timescales. Dodds-Eden et al. (2011) claim evidence for a two-state variability process based on the distribution of flux densities that occurred during VLT measurements in the $K$-band, while Witzel et al. (2012) can explain the distribution of flux densities by a convolution of an intrinsic power-law distribution and a Gaussian white measurement noise, implying a continuous one-state variability process. Meyer et al. (2009) show the importance of the correlation 


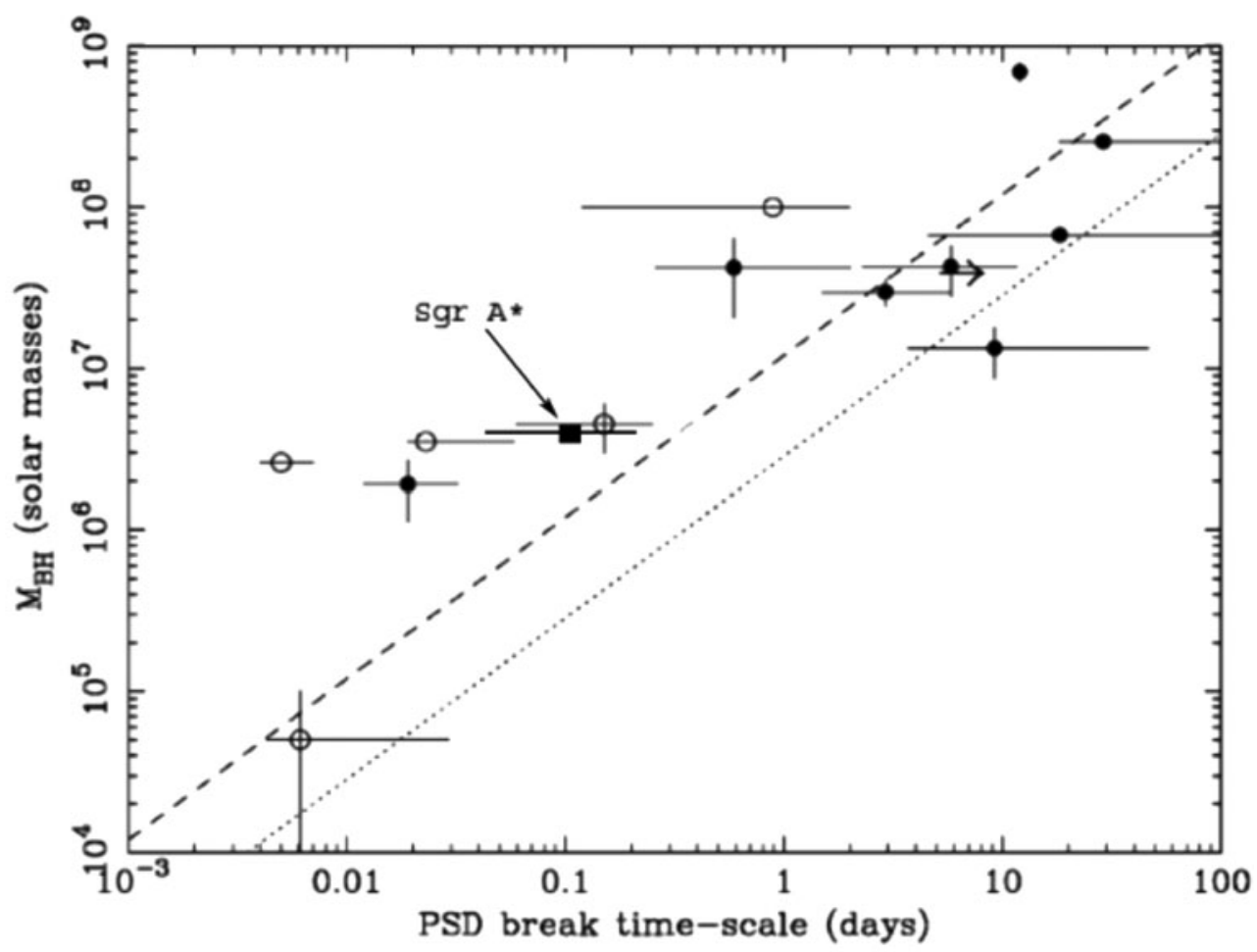

Figure 1. Sgr A*'s break timescale as reported in Meyer et al. (2009) overplotted onto Figure 11 of Uttley et al. (2005), which shows BH mass versus break timescale of the power spectral density for various AGN. The mass of Sgr A* has been taken from Ghez et al. (2008). Its uncertainty corresponds to the height of the black square. Filled circles mark masses determined from optical reverberation mapping, open circles represent masses determined using other methods. The straight lines represent the expected relations if linear mass scaling is assumed from the typical timescales observed in the high/soft (dashed line) and low/hard (dotted line) state of the BHXRB Cyg X-1 (assuming $10 \mathrm{M}_{\odot}$ for its mass).

timescale of the black hole's variability process in the context of other black hole masses, see Figure 1.

The origin of the variable emission has been constrained in multi-wavelength campaigns (see overviews in Genzel et al. 2010 and Morris et al. 2012). Many NIR outbursts have been accompanied by X-ray flares, and every bright X-ray flare that has had synchronous NIR coverage has been accompanied by NIR variability. To explain the correlation between the NIR and the X-rays, pure synchrotron mechanisms, inverse Compton mechanisms and synchrotron self-Compton models have been proposed (Genzel et al. 2010). Other synchronous observations suggest a 1.5-2 hour delay of outbursts between NIR and sub-mm wavelengths, interpreted in the framework of adiabatic expansion of the emitting region, while recent mid-infrared observations resulted in no detection of variable emission (Schödel et al. 2011). An attempt to explain a larger sample of correlated NIR, sub-mm and X-ray variability was presented by Eckart et al. (2012), who favor synchrotron self-Compton models.

The spectral index within the NIR has been the subject of a number of publications (Table 1 ). The derived values cover a range from steep indices of -4 and -2.6 to typical values for optically thin synchrotron radiation around -0.6. Furthermore, Gillessen et al. (2006) claimed a correlation between the spectral index and the flux density, in 
Table 1. Spectral index measurements in the literature.

\begin{tabular}{|c|c|c|}
\hline paper & | spectral index value | & method \\
\hline Eisenhauer et al. (2005)| & $-4 \pm 1$ & SINFONI spectrum \\
\hline Krabbe et al. (2006) & $-2.6 \pm 0.9$ & OSIRIS spectrum \\
\hline Ghez et al. (2005) & $-0.5 \pm 0.3$ & I two broadband filters at flare peak ( $L$ - and $K$-band) \\
\hline Hornstein et al. (2007) | & $-0.6 \pm 0.2$ & | broadband measurements $\mathrm{H}, \mathrm{K}, \mathrm{L}$ (filter cycling), L-M \\
\hline Gillessen et al. (2006) & $\begin{array}{c}-0.6 \pm-0.2 \\
(\operatorname{dim}-1.7 \pm-0.4)\end{array}$ & SINFONI spectrum \\
\hline Bremer et al. (2011) | & $-0.6 \pm 1.6$ & statistical analysis of flare peak fluxes \\
\hline Trap et al. (2011) & $-0.4 \pm 0.3$ & $L$ - and $K$-band filter cycling \\
\hline
\end{tabular}

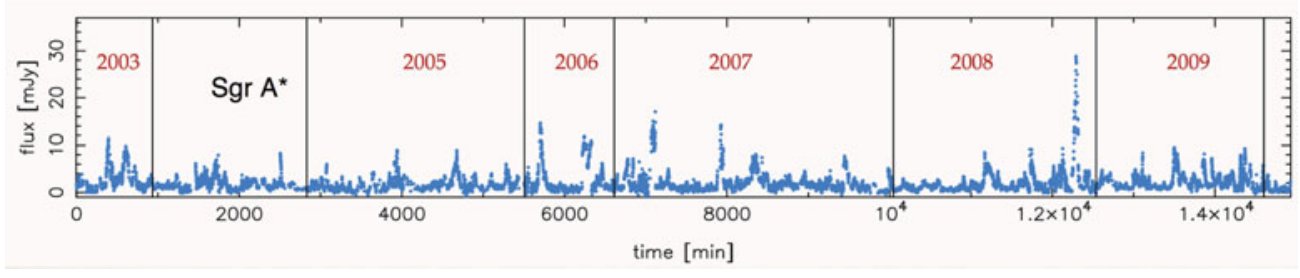

Figure 2. The concatenated light curve of Sgr A* with time gaps longer than 30 minutes removed. [A COLOR VERSION IS AVAILABLE ONLINE.]

contradiction to Hornstein et al. (2007) who found it to be independent of the flux density.

\section{The statistics of the variability process}

In Witzel et al. (2012) we present the currently most comprehensive, consistent $K$-band light curve from NACO (VLT) observations between 2003 and 2010. The data are shown in Figure 2. For convenience we show a concatenated light curve without observation gaps longer than 30 minutes. This visualization shows the data of all nights as a pseudocontinuous light curve allowing for a comparison of the variability and the confusion levels within the individual epochs. A visualization of the true cadence is presented in Figure 3 .

\subsection{Flux density distribution}

A primary result of our statistical analysis is that a power-law distribution is a sufficient description of the intrinsic flux density distribution insofar as it is constrained by the current measurements. The probability density of a power-law distribution is defined as:

$$
p(x)=\left\{\begin{aligned}
0 & : x \leqslant x_{\min }+x_{0} \\
\frac{\alpha-1}{x_{\min }} \cdot\left(\frac{x-x_{0}}{x_{\min }}\right)^{-\alpha} & : \quad x>x_{\min }+x_{0},
\end{aligned}\right.
$$

with $x_{\text {min }}=x_{\text {min,intr }}-x_{0}$ and $x_{\text {min,intr }}$ the lowest value to which the data is power-law distributed, making the power-law normalizable with normalization factor $(\alpha-1) \cdot x_{\min }^{\alpha-1}$, and $x_{0}$ the pole of the power-law. We find:

$$
\begin{aligned}
x_{0} & =(-2.94 \pm 0.1) \mathrm{mJy} \\
x_{\min } & =(4.22 \pm 0.1) \mathrm{mJy} \\
\alpha & =(4.215 \pm 0.05) .
\end{aligned}
$$




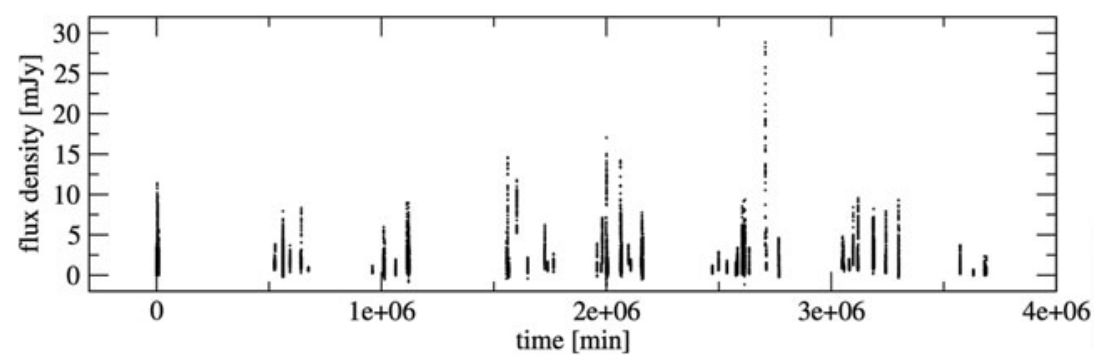

Figure 3. Light curve of Sgr $\mathrm{A}^{*}$ as in Figure 2, but in this case no time gaps have been removed; the true time coverage of the data is shown. Only about $0.4 \%$ of the 7 years have been covered by observations.

This is a valid description for flux densities higher than $x_{\min }$.

We can show that our data sample is consistent with a pure power-law describing the intrinsic flux density distribution under the influence of an instrument with limited resolution and sensitivity and that $x_{\min }$ can be interpreted as the detection limit of $\mathrm{NACO}$ for Sgr A* due to being embedded in extended flux and its confusion by faint unresolved stars. The argument is simple: if we weight the power-law distribution for fluxes higher than $x_{\min }$ with a factor $n_{\text {tail }} / n$, with $n_{\text {tail }}$ the number of data points with values higher than $x_{\min }$ and $n$ the total number of points, we can extend the power-law to smaller flux densities until its integral becomes unity:

$$
x_{\min }^{*}=\left(\frac{n_{\text {tail }}}{n}\right)^{\frac{1}{\alpha-1}} \cdot x_{\min } .
$$

In our case with the values of Equation (2.2) we find $x_{\mathrm{min}}^{*}=3.57 \pm 0.1 \mathrm{mJy}$. Correcting this value for $x_{0}=-2.94 \pm 0.1 \mathrm{mJy}$, this power-law distribution shows a cut-off at $x_{\min }^{*}+x_{0}=0.63 \pm 0.15$, which is identical to the average flux density of background apertures close to Sgr A*. The measured distribution now can be obtained by convolving the power-law distribution that has been extrapolated to small flux densities with a Gaussian distribution to account for the uncertainty of the photometry (see Figure 4).

We conclude that a single continuous variability state and instrumental white noise are sufficient ingredients to describe the observed flux density distribution. The instrumental effects on the photometry are sufficiently described by a Gaussian distribution centered around the typical background value of $0.6 \pm 0.1 \mathrm{mJy}$ with a constant $\sigma=0.32 \mathrm{mJy}$. This instrumental effect leads to a detection limit, which here is defined as the limit up to which reliable photometry is not possible, $\sim 0.7 \pm 0.16 \mathrm{mJy}$ intrinsically, and $\sim 1.3 \pm 0.15 \mathrm{mJy}$ for the actual measurements, which include the background flux density of $0.63 \pm 0.15 \mathrm{mJy}$. With the power-law model we find a median value for the flux density of $\operatorname{med}(x)_{\text {intr. }}=0.9 \pm 0.15 \mathrm{mJy}$ (corrected for the background), or $\operatorname{med}(x)_{\text {obs. }}=1.5 \pm 0.1 \mathrm{mJy}$ (including the background flux density). This shows that, assuming we can extrapolate the power-law to smaller flux densities below the detection limit, we find the median flux density to be very close to the detection limit.

\subsection{RMS-flux density relation}

For the study of X-ray binary variability (linear) RMS-flux relations represent an important piece of information. A relation between RMS and the radiation flux was initially discovered in observations of black-hole and neutron star X-ray binaries (Uttley \& McHardy 2001). Since then the RMS-flux relation has been studied in several other 

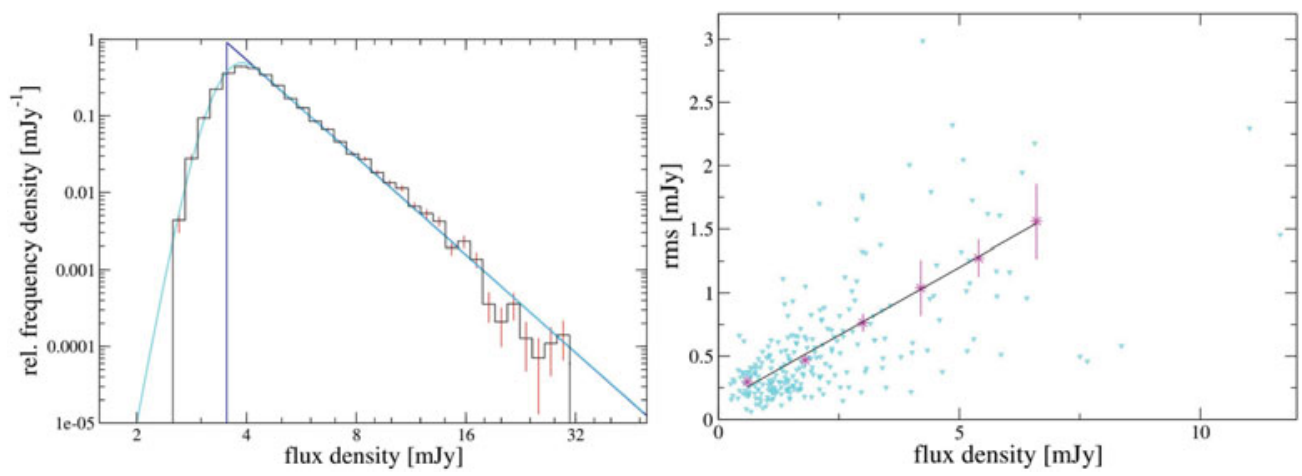

Figure 4. Left: Flux density histogram. The blue line shows the extrapolation of the best power-law fit, the cyan line the power-law convolved with a Gaussian distribution with $\sigma=0.32$ mJy. Right: RMS-flux relation for the variability of Sgr A*. In cyan the actual measurements, in magenta the re-binned data and in black the best linear fit. [A COLOR Version IS AVAILAble online.]

observations of black-hole binaries, active galactic nuclei, neutron-star X-ray binaries, and ultra-luminous X-ray sources (ULX) (see e.g. Heil et al. 2011, and references cited therein). The RMS (root mean square) - as it is used here - is a measure for the magnitude of the variability of the light curve. Following Uttley et al. (2005) the absolute RMS amplitude of variability $\sigma_{\mathrm{RMS}}$ of a time series of $n$ data points, $x_{i}$, is defined as:

$$
\sigma_{\mathrm{RMS}}=\sqrt{\frac{1}{n-1} \sum_{i=1}^{n}\left(x_{i}-\langle x\rangle\right)^{2}} .
$$

In the case of weakly non-stationary segments of a stationary light curve, $\sigma_{\mathrm{RMS}}$ varies randomly about a mean value. Under certain circumstances this mean scales with the average flux density of the segment $\langle x\rangle$.

In particular, Uttley et al. (2005) related the RMS-flux relation (which could be observed on all timescales for some of their sources) to a formally non-linear, in their case exponential, statistical model. With this model the authors can convincingly reproduce the behavior of the observed X-ray light curves and rule out additive (shot-noise) models or self-organized criticality as the responsible processes. They conclude that the variability processes must be multiplicative. Because the RMS-flux relation is stable for all spectral states of the black hole X-ray binary Cyg X-1, independent of its PSD shape, it is believed to be a more fundamental property of the variability than the PSD shape.

In Witzel et al. (2012) we report the discovery of the RMS-flux relation for Sgr A* in the NIR. Following the description of the RMS analysis in Uttley et al. (2005) and, using time series IDL-codes written by S. Vaughan, we estimate the flux density dependency of the RMS on a frequency range from the average Nyquist-frequency of $\sim 0.5 \mathrm{~min}^{-1}$ to $0.06 \mathrm{~min}^{-1}$ (corresponding to timescales of 2 to 16 minutes) for data sections with a length of $24 \mathrm{~min}$. The algorithm works as follows: we divided the light curves into continuous data segments of 24 minutes, took the average as a flux density estimate, and determined the PSD of this section. We then obtained the RMS for timescales between 2 and 16 minutes by taking the square root of the integral of the PSD over the corresponding frequency range.

The dominant timescale is of the order of a few hundred minutes (150 min, Meyer et al. 2009). Realizations of the random process that are not significantly longer than this timescale are weakly non-stationary. So for a given mean flux density the 


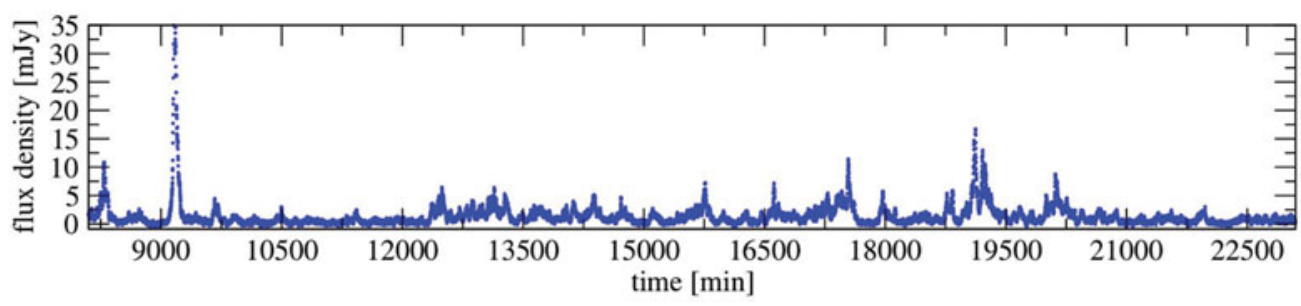

Figure 5. Simulated lightcurves taken from a $4 \cdot 10^{6}$ min time series created with the best double-broken power-law PSD from Witzel et al. (2012), showing typical $15000 \mathrm{~min}$ of variability. [A COLOR VERsion is AVAILAbLE ONLINE.]

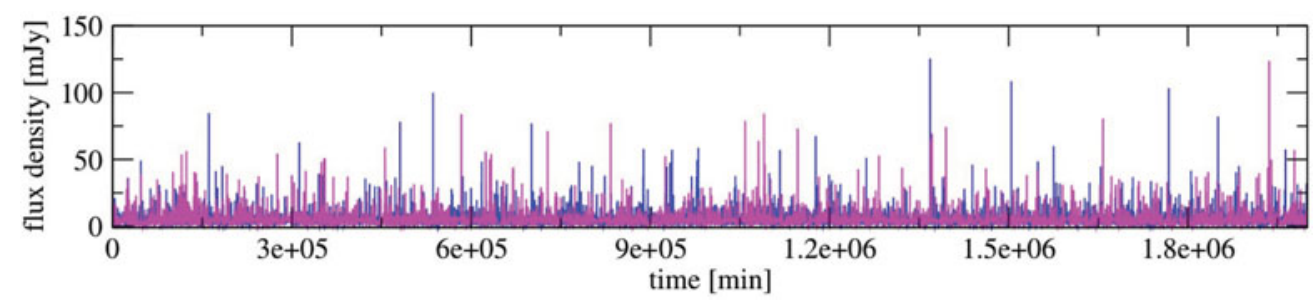

Figure 6. Long-term appearance of light curves generated with the best single (blue) and the best double (magenta) broken PSD (from Witzel et al. 2012). [A COLOR Version is AVAilable online.]

RMS-values of our data sections of 24 minutes are significantly scattered around their average value, and we re-binned the obtained RMS-values into flux density-bins with a width $\Delta F=1.2 \mathrm{mJy}$. The result is shown in Figure 4 . To a first approximation, we find a linear RMS-flux dependence.

\subsection{Simulations}

Based on a transformation derived in Witzel et al. (2012) we are able to generate surrogate light curves for any input power spectral density (PSD) that show the observed flux density distribution. We repeat the recipe here that allows us to simulate long and continuous light curves that are typical for what has been observed:

- Generate a Gaussian, linear light curve following the method by Timmer \& Koenig (1995), i.e., drawing Fourier coefficients for each frequency from a Gaussian distribution with a variance proportional to the value of the PSD at that frequency, and Fourier transforming to the time domain.

- Normalize the obtained Gaussian process to a variance of unity. Optionally re-sample the equally spaced data to the cadence of the observed data.

- Transform the light curve according to eq. (28) in Witzel et al. (2012); this formula transforms a Gaussian random variable with unit variance to a random variable distributed according to the power-law described above.

- Add an independently drawn quantity (e.g. Gaussian) for each time point to account for the white noise contribution of the measurement.

For the best PSD found in Witzel et al. (2012) we show typical results in Figure 5 and 6 . This algorithm is useful for anybody who intends to plan observations, test measurement errors in variability data or wants to compare the variability at other wavelengths statistically with the NIR variability. In particular, this method maintains the flux density distribution and adapts the normalization of the PSD for any given shape, which is an important prerequisite for comparing absolute values of structure functions in timing analysis. 

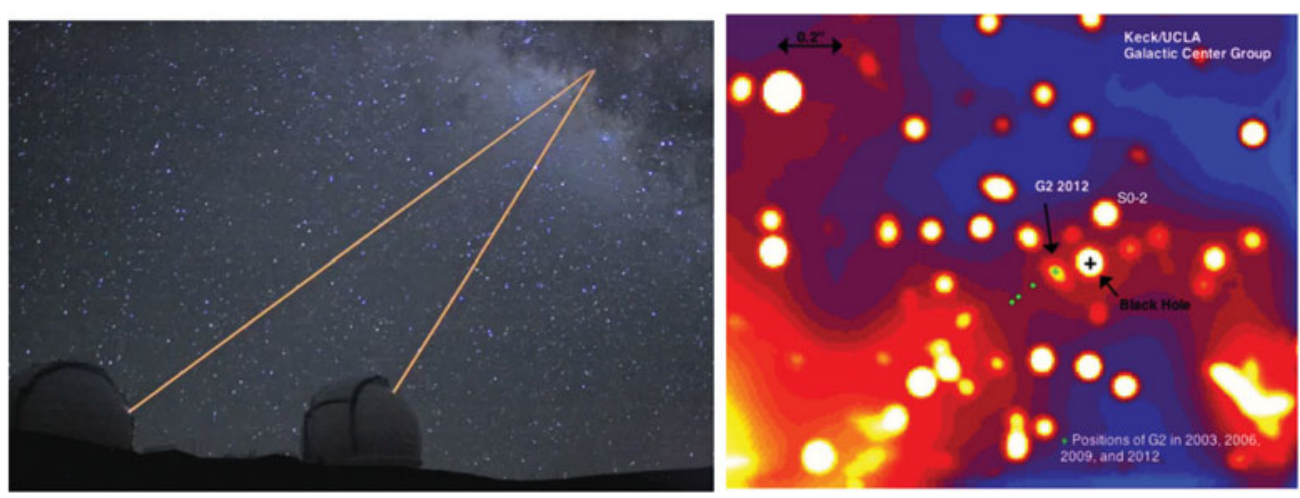

Figure 7. Left: Simultaneous observations with both Keck telescopes with both adaptive optics lasers pointing to the Galactic center (image courtesy: Dan Birchall). Right: Bright flare in both nights of our monitoring. In this combined map the average flux over both nights of Sgr A* appears to be even brighter than the nearby star S0-2. [A COLOR VERSion IS AVAILABLE ONLINE.]

\section{A new measurement of the spectral index}

In July 2012 the Galactic center group at UCLA conducted a spectral index experiment in the NIR with both Keck telescopes, using the NIR imager NIRC2 at Keck II in $L^{\prime}$-band and the imager of OSIRIS at Keck I in $H$-band in order to measure for the first time the spectral index development during a bright flare with synchronous high cadence observations. For two nights we monitored Sgr A* and observed in both nights extraordinarily bright flares (see Figure 7). In $L^{\prime}$-band we reached a cadence of 20 sec in both nights, and in $H$-band $50 \mathrm{sec}$ and $30 \mathrm{sec}$, respectively. This cadence resulted in stretches of data that has been observed simultaneously within about $10 \mathrm{sec}$, on average. The accuracy of the time stamps of the data were checked carefully with timing tests based on the exact clocking of the AO-telemetry system. We reduced the data analogously to what is described in Witzel et al. (2012) and conducted aperture photometry on deconvolved images. We used several constant stars as relative calibrators and dereddened the flux density according to the extinction law given by Schödel et al. (2010).

The resulting light curves are shown in Figure 8 together with a number of constant calibrators to illustrate the high accuracy of the relative calibration. In these plots we present a version of the $L^{\prime}$-band data (red) that has been scaled to $H$-band with the median ratio between $L$ - and $H$-band over all synchronous measurements (independently for nights 1 and 2). As other publications point out (see Table1), a correct treatment of the background is essential in order to derive proper ratios and consequently proper spectral indices. We corrected the background prior to the deconvolution for the first night by subtracting the background estimate with Starfinder (Diolaiti et al. 2000), whereas the data quality of the second night didn't allow a reliable background estimation from images. We then fitted the $L^{\prime}$-band data to the $H$-band data with a constant ratio and an offset as free parameters in a least square minimization. This resulted in an offset value for the first night consistent with zero, indicating a proper background correction before deconvolution, and in a value of $3.4 \mathrm{mJy}$ for the second night. The values for the median ratio in both nights are consistently 0.6 , implying a consistent and correct background treatment for both nights.

The derived spectral indices are plotted versus the corresponding ( $L^{\prime}$-band) flux densities in Figure 9. The scale of the y-axis has been chosen according to Hornstein et al. (2007) and Gillessen et al. (2006). Our results show no indication of a correlation between flux density and spectral index and are in full agreement with Hornstein et al. (2007). 

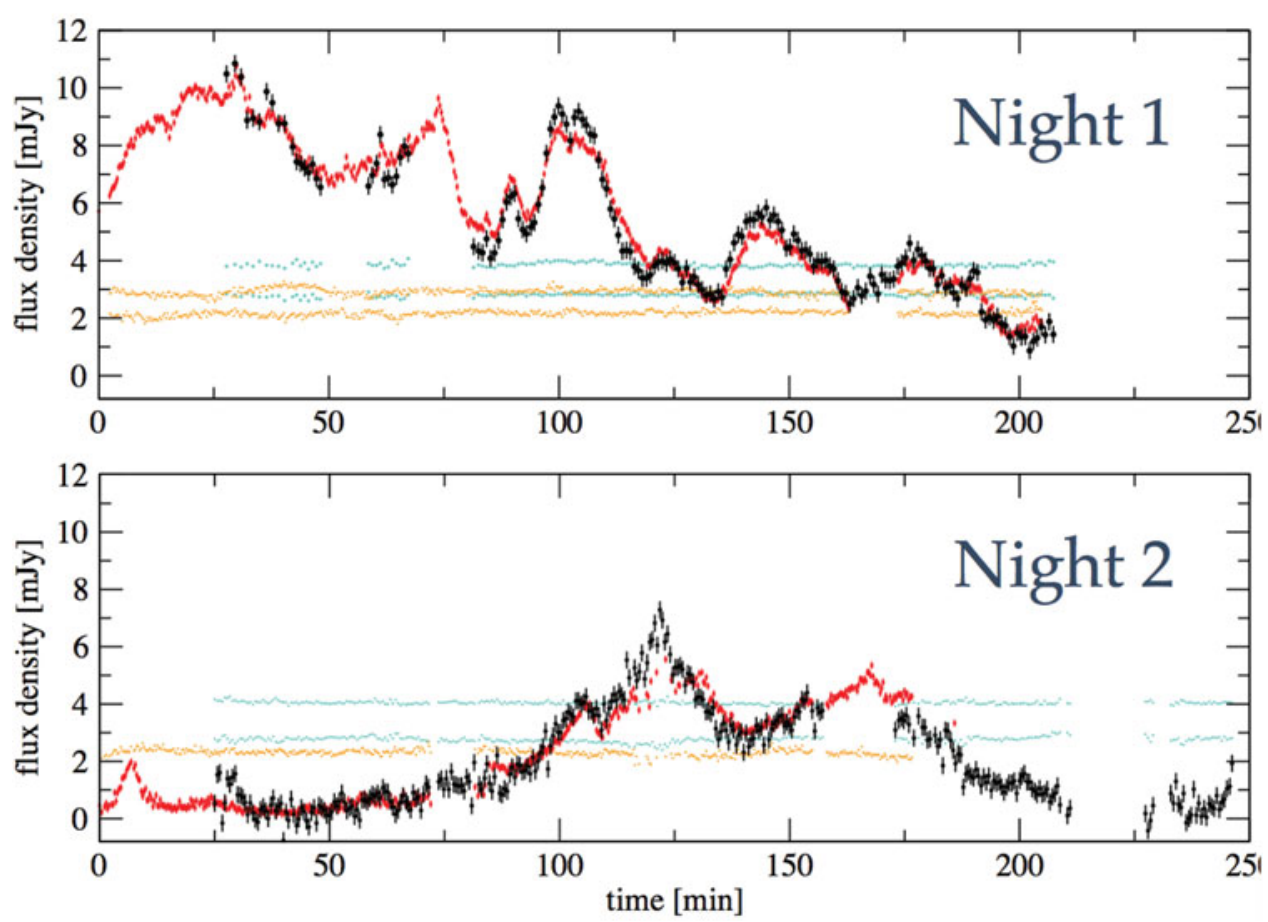

Figure 8. The simultaneous lightcurves of both nights, $L^{\prime}$-band data (red) scaled to $H$-band (black) after reddening correction. Additionally we show calibrator lightcurves for both nights and bands ( $L^{\prime}$-band: orange, $H$-band: cyan). [A Color version is AVAlLable online.]
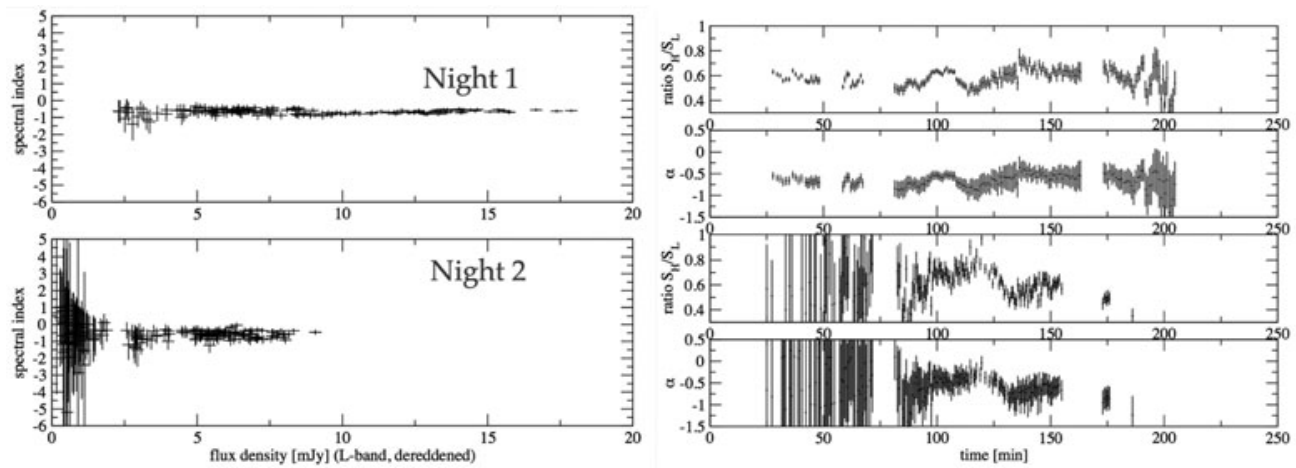

Figure 9. Left: Spectral index versus flux density for both nights. Right: Flux density ratio and spectral index as a function of time.

The spectral index is constant over a wide range of flux densities with values slightly varying around 0.6 in both nights, indicating optically thin synchrotron radiation.

Figure 9 shows the ratio and the spectral index as a function of time. The spectral index is mildly variable. Whether these variations can be further interpreted, e.g. in the framework of a time delay between both wavelengths, is subject to ongoing statistical investigations. 


\section{Conclusions}

- Sgr A* seems to be continuously variable in the near-infrared.

- It shows a linear RMS-flux relation and a break time-scale at a few hours.

- We developed a statistical model to simulate the NIR variability.

- The $L-H$ spectral index is constant with flux and in first order with time at $\alpha=$ $-0.62 \pm 0.1$

\section{References}

Bremer, M., Witzel, G., Eckart, A., et al. 2011, A\& A 532, A26

Diolaiti, E., Bendinelli, O., Bonaccini, D., et al. 2000, in Presented at the Society of PhotoOptical Instrumentation Engineers (SPIE) Conference, Vol. 4007, Proc. SPIE Vol. 4007, p. 879-888, Adaptive Optical Systems Technology, Peter L. Wizinowich; Ed., ed. P.L. Wizinowich, 879-888

Do, T., Ghez, A. M., Morris, M. R., et al. 2009, ApJ 691, 1021

Dodds-Eden, K., Gillessen, S., Fritz, T. K., et al. 2011, ApJ 728, 37

Eckart, A., García-Marín, M., Vogel, S. N., et al. 2012, A\& A 537, A52

Eckart, A., Schödel, R., Meyer, L., et al. 2006, A\&A 455, 1

Eisenhauer, F., Genzel, R., Alexander, T., et al. 2005, ApJ 628, 246

Genzel, R., Eisenhauer, F., \& Gillessen, S. 2010, Reviews of Modern Physics, 82, 3121

Genzel, R., Schödel, R., Ott, T., et al. 2003, Nature 425, 934

Ghez, A. M., Hornstein, S.D., Lu, J. R., et al. 2005, ApJ 635, 1087

Ghez, A. M., Salim, S., Weinberg, N. N., et al. 2008, ApJ 689, 1044

Gillessen, S., Eisenhauer, F., Quataert, E., et al. 2006, ApJ Lett. 640, L163

Heil, L. M., Vaughan, S., \& Uttley, P. 2011, MNRAS 411, L66

Hornstein, S. D., Matthews, K., Ghez, A. M., et al. 2007, ApJ 667, 900

Krabbe, A., Iserlohe, C., Larkin, J. E., et al. 2006, ApJ Lett. 642, L145

Meyer, L., Do, T., Ghez, A., et al. 2009, ApJ Lett. 694, L87

Meyer, L., Schödel, R., Eckart, A., et al. 2006, A\& A 458, L25

Morris, M. R., Meyer, L., \& Ghez, A. M. 2012, Research in Astronomy and Astrophysics, 12, 995

Schödel, R., Morris, M. R., Muzic, K., et al. 2011, A\&A 532, A83

Schödel, R., Najarro, F., Muzic, K., \& Eckart, A. 2010, A\&A 511, A18

Timmer, J. \& Koenig, M. 1995, A\&A 300, 707

Trap, G., Goldwurm, A., Dodds-Eden, K., et al. 2011, A\&A 528, A140

Uttley, P. \& McHardy, I. M. 2001, MNRAS 323, L26

Uttley, P., McHardy, I. M., \& Vaughan, S. 2005, MNRAS 359, 345

Witzel, G., Eckart, A., Bremer, M., et al. 2012, ApJS 203, 18 